\title{
Convergence sur l'apprenant et ouverture sur une société en mutation
}

\section{Françoise Haramboure}

\section{CpenEdition}

\section{Journals}

Édition électronique

URL : http://journals.openedition.org/asp/3924

DOI : 10.4000/asp.3924

ISSN : 2108-6354

\section{Éditeur}

Groupe d'étude et de recherche en anglais de spécialité

\section{Édition imprimée}

Date de publication : 1 décembre 1995

Pagination : $277-283$

ISSN : 1246-8185

\section{Référence électronique}

Françoise Haramboure, « Convergence sur l'apprenant et ouverture sur une société en mutation », ASp [En ligne], 7-10 | 1995, mis en ligne le 30 septembre 2013, consulté le 08 mai 2019. URL : http:// journals.openedition.org/asp/3924; DOI : 10.4000/asp.3924

Ce document a été généré automatiquement le 8 mai 2019.

Tous droits réservés 


\title{
Convergence sur l'apprenant et ouverture sur une société en mutation
}

\author{
Françoise Haramboure
}

1 Les concepts de centration sur l'apprenant et d'ouverture interculturelle sont à première vue divergents. Le premier se réfère à l'acteur central de l'apprentissage, le second à son environnement. L'objet de cet article est de mettre en évidence les convergences existant entre ces deux concepts dans l'acquisition de la compétence de communication en langue étrangère dans le cadre des filières technologiques de l'enseignement supérieur.

\section{L'approche centrée sur l'apprenant}

2 Par delà les effets de mode, l'approche centrée sur l'apprenant engage un changement de perspective. Elle implique une bifocalisation de l'enseignement/apprentissage qui ne se préoccupe plus seulement des savoirs et savoir-faire à maîtriser en langue cible, mais également de la construction de ces derniers par l'apprenant.

3 Elle résulte de la prise en compte de deux types de données convergentes:

4 - d'une part, l'observation sur le terrain par les enseignants que les actes d'enseignement ne se transforment pas automatiquement en actes d'apprentissage. Cette constatation a entraîné une réévaluation du rôle joué par l'apprenant dans ce processus.

5 - d'autre part, la confirmation de ces observations par les résultats des recherches en psychologie cognitive, c'est à dire la mise en évidence que l'acquisition résulte essentiellement de l'activité de l'apprenant. Autrement dit, l'apprentissage est fonction de la capacité de ce dernier à apprendre.

6 Elle reflète également une préoccupation double: celle de permettre à l'étudiant spécialiste d'autres disciplines de mieux exploiter le temps nécessairement limité imparti à la formation linguistique, par la mise en œuvre de tout son potentiel d'apprenant et 
celle de lui donner les outils nécessaires à la poursuite de l'apprentissage, en autonomie, hors formation.

Dans cette perspective, l'un des objectifs de l'enseignement/apprentissage est de développer la capacité de l'individu d'apprendre à savoir, d'apprendre à apprendre.

De ce fait, il ne suffit pas de procéder à une analyse des publics concernés et de leurs besoins linguistiques en fonction de la finalité de leurs formations pour définir les objectifs de l'enseignement. La reconnaissance du rôle de l'apprenant dans l'apprentissage implique que l'on intègre dans cette définition trois paramètres majeurs : ses représentations de l'apprentissage; son style d'apprentissage; les opérations mentales sous-jacentes aux capacités sur lesquelles repose la compétence de communication en langue étrangère.

\section{Les représentations de l'apprentissage linguistique}

9 En effet, si l'apprenant est l'acteur essentiel de l'apprentissage, il convient en premier lieu d'élucider son attitude et ses attentes à cet égard.

10 Afin de procéder à leur analyse, nous nous sommes appuyée sur les observations recueillies en cours de langue auprès de deux promotions d'étudiants et sur les réponses au questionnaire élaboré par E. Horwitz intitulé «Beliefs about Language Learning» (1987: 127-128).

11 Issus des filières scientifiques et techniques des lycées où l'anglais est considéré comme une discipline secondaire, les étudiants montrent un intérêt limité pour cette matière en début de formation initiale à l'université. Cette attitude se manifeste par leur faible investissement dans les activités proposées en cours et une réticence à s'exposer à toute pratique de la langue en dehors de ce cadre. Elle est associée à une image négative de leur compétence linguistique dont témoigne leur appréhension des premiers tests.

12 Les réponses au questionnaire corroborent ces observations. L'apprentissage d'une langue étrangère est considéré par $70 \%$ des étudiants comme un apprentissage distinct des autres apprentissages relevant d'une aptitude particulière " a special ability». D'une part, cette appréciation est le reflet de l'opinion communément répandue selon laquelle l'acquisition d'une langue étrangère relève d'un don; elle révèle également que les étudiants ne sont pas conscients des opérations sur lesquelles se fondent les tâches de compréhension ou de production en langue cible. D'autre part, elle laisse supposer qu'ils ne font pas appel, dans ce cas, aux capacités d'analyse et de synthèse sollicitées par l'apprentissage d'autres disciplines telles que les mathématiques et la physique.

13 L'analyse de leurs stratégies d'apprentissage confirme cette dernière interprétation. La priorité est accordée par la majorité des étudiants à l'étude de la grammaire et du vocabulaire. Dans ces circonstances, il est cohérent que la répétition soit parmi leurs stratégies d'apprentissage privilégiées. Cette attitude n'est pas sans intérêt : elle confirme notamment l'hypothèse que tout un pan du potentiel d'apprentissage de l'apprenant n'est pas exploité notamment ses facultés d'analyse et de synthèse. Elle est sans doute responsable de leur insatisfaction lorsque, après avoir tenté de mémoriser lexique et règles de grammaire pendant de nombreuses années, ils ne parviennent encore pas à effectuer des tâches de compréhension et d'expression. 
14 En effet, pour la plupart des étudiants concernés, comprendre un énoncé oral ou écrit implique essentiellement le décodage de chacun des items lexicaux utilisés. L'analyse des diverses composantes $\mathrm{du}$ contexte, leurs connaissances sont négligées dans la construction du sens. Les insuffisances de ces procédures sont évidentes lorsqu'ils sont exposés à des documents scientifiques relevant de leurs spécialités. Ils se privent alors de l'apport de leurs savoirs dans le domaine qui faciliterait la compréhension de l'énoncé.

15 S'agissant de l'expression, les étudiants de niveau faible s'appuient essentiellement sur la traduction L1-L2. Ignorant les procédures sur lesquelles se fonde la production d'un message, ils pratiquent spontanément le transfert lexical et morpho-syntaxique entre langue maternelle et langue étrangère. Il en résulte la fixation dans leur interlangue de formes nativisées du type "*I'm agree with you» et des productions incohérentes négligeant la visée de l'échange.

Leur représentation de l'évaluation mérite également réflexion. Leur difficulté à porter un jugement sur leurs performances ou sur celles de leurs pairs est révélatrice à plusieurs égards: ils considèrent qu'il s'agit d'une opération relevant exclusivement de la compétence de l'enseignant. Leurs lacunes en matière de connaissances procédurales les empêchent de procéder à l'évaluation de leurs performances.

17 Enfin, la dissociation par les étudiants des concepts de langue et culture est au cœur même de notre problématique. Elle repose sur une perception de la langue envisagée comme un système indépendant de son contexte social et culturel d'utilisation. Elle révèle également une conception réductrice de la culture, étrangère aux pratiques de la vie quotidienne et aux valeurs qui les sous-tendent. Elle renvoie enfin à une perception lettrée de la culture qui exclut de son champ les domaines qui leur sont familiers de la science et la technique.

\section{La mise en œuvre de la démarche}

Compte tenu des limites de cette vision de l'apprentissage, il importe que l'enseignement de la langue étrangère intègre parmi ses objectifs prioritaires le développement des procédures d'apprentissage des apprenants. La satisfaction de cet objectif suppose plusieurs étapes :

\section{Une phase préliminaire}

Il s'agit de prendre en compte l'existant, c'est-à-dire les moyens que les apprenants utilisent consciemment ou non pour apprendre. Au cours de cette étape, les étudiants sont amenés, à l'aide d'un questionnaire élaboré dans ce but, à reconnaître les démarches et les outils auxquels ils ont recours.

Plus concrètement, l'apprenant détermine notamment s'il a tendance à adopter une démarche analytique ou globaliste ; les modes perceptuels qu'il utilise habituellement, le mode visuel ou auditif lorsqu'il est par exemple confronté à la compréhension d'un support vidéo authentique ; son attitude à l'égard de la prise de risques. 


\section{Des pauses méthodologiques}

21 Cette sensibilisation de l'apprenant à son profil personnel d'apprentissage reste sans effet si elle n'est pas directement associée aux activités d'apprentissage d'une langue étrangère et à leurs contraintes spécifiques. Dans cette optique, l'intégration de pauses méthodologiques s'inscrivant directement dans le prolongement des tâches de compréhension et de production s'avère pertinente. Elles offrent la possibilité à l'étudiant d'expliciter les procédures adoptées pour les effectuer, d'identifier les problèmes rencontrés et d'en analyser les causes avec l'aide de l'enseignant.

Les tâches de compréhension écrites ou orales sont l'occasion pour les étudiants analytiques de repérer les limites de l'utilisation quasi exclusive de l'analyse des formes verbales au détriment du recours au contexte. Inversement, elles conduisent les étudiants globalistes à saisir l'intérêt de l'analyse formelle s'ils veulent parvenir à une compréhension approfondie du document en langue cible.

Les tâches d'expression écrites ou orales contribuent également à leur faire découvrir l'importance de prendre en compte les phases de conception et de structuration du message pour qu'il réponde à la finalité de l'échange.

\section{Des tâches d'apprentissage orientées vers la mise en œuvre de procédures pertinentes}

Parallèlement, les tâches d'apprentissage sont conçues par l'enseignant de façon à favoriser la mise en œuvre des procédures spécifiques à chacune des activités.

À cet effet, en matière de production, l'élaboration de consignes claires définissant le but de l'interaction, imposant l'utilisation de marqueurs de liaisons pour en assurer la cohérence voire de formes verbales correspondant à la thématique de l'énoncé est une condition indispensable. Elles font appel aux diverses opérations mentales responsables de la production d'un message en langue étrangère, à savoir la conception du message, sa structuration et la mobilisation de formes adéquates.

S'agissant de compréhension, des grilles de questions facilitent le développement de procédures adéquates et leur gestion en fonction du type de compréhension attendue. S'il s'agit d'une activité de compréhension globale, les questions porteront essentiellement sur les composantes contextuelles. Pour une tâche de compréhension approfondie, elles feront appel alternativement aux données contextuelles et à l'analyse des formes rencontrées.

Fondées sur les mêmes préoccupations, les tâches de communication intégrée telles que les jeux de rôle visent à mobiliser les procédures alternativement responsables de la production et de la compréhension exposées précédemment. Elles ont aussi pour but de développer chez les étudiants des stratégies de coopération, demandes de clarification, reformulations et définitions dont dépend la réussite des échanges. La mise en œuvre des stratégies de coopération présente un intérêt double : d'une part, elle conduit le locuteur à faire appel à d'autres ressources en langue cible pour faciliter la compréhension de son partenaire et soumet ce dernier à un nouveau travail de construction du sens; d'autre part, elle permet aux étudiants d'acquérir les outils qui leur permettront de surmonter les difficultés inhérentes aux situations de communication exolingue. Ce faisant, 
l'étudiant perfectionniste est amené par l'expérience à utiliser la paraphrase s'il ne veut pas bloquer l'échange par la recherche du mot juste et celui qui prend naturellement des risques à se concentrer sur les formes s'il veut que son énoncé soit compris par son interlocuteur.

Progressivement, ce guidage procédural devient superflu. Les étudiants parviennent à sélectionner les procédures adéquates de leur propre initiative.

\section{Une réflexion systématique sur la relation forme-sens}

Dans ce cadre, il appartient à l'enseignant de souligner l'intérêt d'une contrainte procédurale constitutive de l'apprentissage d'une langue étrangère à savoir la réflexion systématique sur la relation forme-sens par des exercices spécialement prévus dans ce but. À ce titre, par exemple, la distinction entre present perfect et simple past, difficile à stabiliser pour les étudiants, est mieux conceptualisée s'ils effectuent l'analyse comparée des deux formes verbales en contexte et en déduisent la signification à partir des indices fournis. En l'absence de cette procédure, les étudiants procèdent par tâtonnement ou pratiquent le transfert à partir de leur langue maternelle. En l'occurrence, ils font l'amalgame entre present perfect et passé composé. Sans ce retour réflexif sur les formes rencontrées au cours des activités de compréhension et de production, l'étudiant analytique se voit privé des bénéfices de la démarche qu'il adopte spontanément et les ressources linguistiques de l'étudiant globaliste restent peu assurées.

Par ailleurs, cette procédure facilite la transformation des faits de langue rencontrés de façon éphémère en traces durables dans la mémoire à long terme, c'est-à-dire le passage de l'«input " à l'« intake». Elle contribue à la restructuration des connaissances de l'apprenant en fonction des nouvelles relations établies. Elle favorise la mémorisation sémantique du concept qui ne se construit qu'avec la répétition, comme le souligne A. Lieury (1994) : « la mémorisation sémantique des concepts s'améliore au fur et à mesure du nombre de répétitions d'épisodes contextuels ».

\section{L'évaluation}

31 Dans cette perspective, les phases d'évaluation tant formative que sommative font partie intégrante de cette démarche.

S'agissant d'évaluation formative, elle s'appuie non seulement sur les critères strictement linguistiques de correction lexicale, grammaticale et articulatoire, mais encore sur la pertinence des procédures utilisées. Intervenant à intervalles réguliers dans le processus d'apprentissage, elle a pour fonction d'analyser les problèmes rencontrés par les étudiants en termes de procédures et de rechercher les solutions les plus efficaces en regard de l'attitude de chaque étudiant et de la finalité de la tâche. C'est à cette occasion, par exemple, qu'un étudiant découvrira que ses problèmes en matière d'intonation et de prononciation sont liés à sa tendance à utiliser les informations visuelles au détriment des informations auditives. Dès lors, l'évaluation s'inscrit pleinement dans le processus d'apprentissage. Par un effet de rétroaction, elle permet à l'étudiant d'envisager les mesures correctives les mieux adaptées à son profil cognitif et d'élargir son potentiel d'apprentissage. En ce sens, elle participe directement à l'acquisition par l'étudiant des outils qui lui permettent d'évaluer son propre apprentissage. 
, l'évaluation sommative relève également de cette démarche dans la mesure où elle sanctionne l'acquisition des procédures appropriées aux tâches évaluées au même titre que celles des savoirs linguistiques.

\section{Conclusion}

En conclusion, le dispositif présenté n'a pas la prétention d'être exhaustif. Il constitue une contribution visant l'exploitation par le sujet apprenant de tout son potentiel d'apprentissage et le développement de procédures tenant compte des exigences spécifiques de l'acquisition de la compétence de communication en langue étrangère. Néanmoins, cette approche serait incomplète si elle ne s'accompagnait d'une ouverture sur l'environnement social et culturel de l'apprentissage.

\section{BIBLIOGRAPHIE}

Horwitz, E. K. 1987. « Surveying student beliefs aboutl learning ». In Wenden, Anita et Joan Rubin, Learner Strategies in Language Learning. Londres : Prentice Hall International.

Liuery, A. 1994. « Améliorer sa mémoire. Mythes et réalité ». Sciences Humaines 43.

\section{RÉSUMÉS}

À première vue, les concepts de centration sur l'apprenant et de formation à l'interculturel semblent divergents. Le premier se réfère à l'apprenant, le second à la dimension interculturelle de l'apprentissage linguistique. L'objet de cette communication est de montrer la convergence de ces deux concepts notamment dans l'apprentissage de l'anglais, langue de spécialité.

At first sight, the concepts of learner-centredness and intercultural education seem to diverge. The former refers to the learner and the latter to the learning environment. The aim of this paper is to show how these concepts should converge, especially in the field of ESP.

\section{INDEX}

Mots-clés : anglais de spécialité, centration sur l'apprenant, formation, interculturel Keywords : ESP, learner-centredness, intercultural, training 


\section{AUTEUR}

\section{FRANÇOISE HARAMBOURE}

Françoise Haramboure, maître de conférences à l'Université Bordeaux 3 consacre ses activités d'enseignement et de recherche à la didactique des langues étrangères en formation initiale et continue.f.haramboure@cegetel.net 